\title{
Puntos de equilibrio Y eStabilidad en lazo ABIERTO PARA UN SERVOMECANISMO NEUMÁTICO DE POSICIONAMIENTO
}

\author{
GeRmÁn A. BACCA ${ }^{1}$
}

\section{Resumen}

El comportamiento dinámico de un servomecanismo neumático de posicionamiento es altamente no-lineal debido principalmente a la compresibilidad del aire y a la fricción presente en el elemento actuador. En este artículo se realiza un estudio de la dinámica nolineal de este sistema en lazo abierto mediante la determinación de los puntos de equilibrio, análisis de estabilidad y determinación de bifurcaciones. Para el análisis de estabilidad del sistema se recurre al método de linealización de Lyapunov. Los resultados teóricos para el equilibrio del servosistema se obtienen mediante la aplicación de métodos numéricos y son confrontados con datos experimentales.

\section{Palabras clave}

Modelado matemático, punto de equilibrio, ecuaciones diferenciales no-lineales, estabilidad.

\section{Abstract}

The dynamic behavior of a pneumatic servo positioning system is highly non-linear mainly due to air compressibility and the friction present in the actuator. In this paper, is presented the study

1 Ingeniero Mecánico. Especialista en Informática Industrial. Magíster en Sistemas Automáticos de Producción. Profesor Asociado, Universidad del Cauca, Popayán, gbacca@unicauca.edu.co

Fecha de recepción: 13 de marzo de 2010 Fecha de aceptación: 25 de junio de 2010 
of nonlinear dynamics of this system in open loop, through the determination of the equilibrium points and stability analysis. For the stability analysis of the system uses the Lyapunov's linearization method. The theoretical results of servosystem equilibrium are obtained by applying numerical methods and are compared with experimental data.

\section{Keywords}

Mathematical modeling, equilibrium point, nonlinear differential equations, stability. 


\section{INTRODUCCIón}

En los últimos años, el análisis de servomecanismos neumáticos ha sido retomado por la gran variedad de aplicaciones que ofrecen estos sistemas en el campo de la técnica de manipulación de piezas como: sujeción, desplazamiento, posicionamiento y orientación.

Sin embargo, las ecuaciones básicas de los sistemas neumáticos son no-lineales y tienden a ser más complejas que las que se obtienen en sistemas que trabajan con fluido incompresible. Esto explica por que el estudio en este campo ha recibido hasta hace un corto tiempo poca atención.

La compresibilidad del aire y la fricción son causantes de fenómenos como adherencia-deslizamiento (stick-slip), oscilaciones autoexcitadas o ciclos límites, bifurcaciones e incluso caos en estos tipos de sistemas. Estos fenómenos tienden a generar comportamientos irregulares que son perjudiciales sobre todo en aplicaciones de posicionamiento. Por lo tanto, el diseño de una estrategia de control óptima requiere en primera instancia una identificación y análisis de estos fenómenos no-lineales con el fin de poder compensar sus efectos.

Trabajos de investigación sobre fenómenos no-lineales en sistemas de potencia fluida (neumáticos e hidráulicos) no se encuentran en gran cantidad en la literatura científica como si ocurre en sistemas eléctricos. Algunos de estos trabajos se han realizado mediante investigaciones en las cuales por medio de un modelo matemático y técnicas experimentales se ha logrado detectar la presencia de fenómenos tales como adherencia-deslizamiento, oscilaciones autoexcitadas o ciclos límites y oscilaciones caóticas (Hayashi, 2000; Takahiro \& Manabu, 2000). El estudio del fenómeno stick-slip con el fin de reducir su efecto en actuadores hidráulicos es presentado en (Owen et al., 2003). En (Yaunjay et al., 2008) se presenta un método para el análisis y la atenuación de ciclos límites debido a la fricción en un servosistema hidráulico con presencia de retardo de transporte. La identificación de bifurcaciones tipo Hopf en compresores neumáticos axiales controlados 
en lazo cerrado bajo realimentación de estado lineal es presentada en (Kang et al., 1999), con este trabajo se pretende el diseño de estrategias de control que permitan cumplir con requerimientos en base a las bifurcaciones del sistema. El objetivo de este artículo es determinar por medio de métodos analíticos y numéricos los puntos de equilibrio de un servosistema neumático en lazo abierto, determinar su estabilidad e identificar fenómenos no-lineales.

En este artículo se presenta en la sección 2 el modelo matemático del servosistema. En la sección 3 se presenta el análisis de la dinámica no-lineal del servosistema en lazo abierto mediante la obtención de sus puntos de equilibrio y la estabilidad presente en estos. Además, se analiza en detalle el fenómeno adherenciadeslizamiento y se identifica el tipo de bifurcaciones presentes en el sistema. En la sección 4 se encuentran las conclusiones. La nomenclatura utilizada y las referencias bibliográficas aparecen al final del documento.

\section{Modelo matemático}

El servomecanismo neumático de posicionamiento mostrado en la Fig. 1 está compuesto principalmente por un actuador lineal de doble efecto tipo Festo DGPL-25-500-PPV-A como elemento de trabajo y por una válvula proporcional de vías tipo Festo MPYE-51/8-010-B como elemento de control. El modelo matemático de este sistema se desarrolló considerando las siguientes suposiciones: El fluido de trabajo es aire y se considera como un gas ideal, se aplica la ley de conservación de la masa en cada volumen de control, se desprecian pérdidas de presión por fricción en los conductos de aire, y las propiedades como la densidad, la temperatura y la presión del aire se consideran uniformes en los diferentes volúmenes de control.

El modelo matemático está descrito básicamente por tres ecuaciones diferenciales no-lineales: una ecuación que describe la dinámica del émbolo y dos ecuaciones que describen la dinámica de la presión en el sistema. 


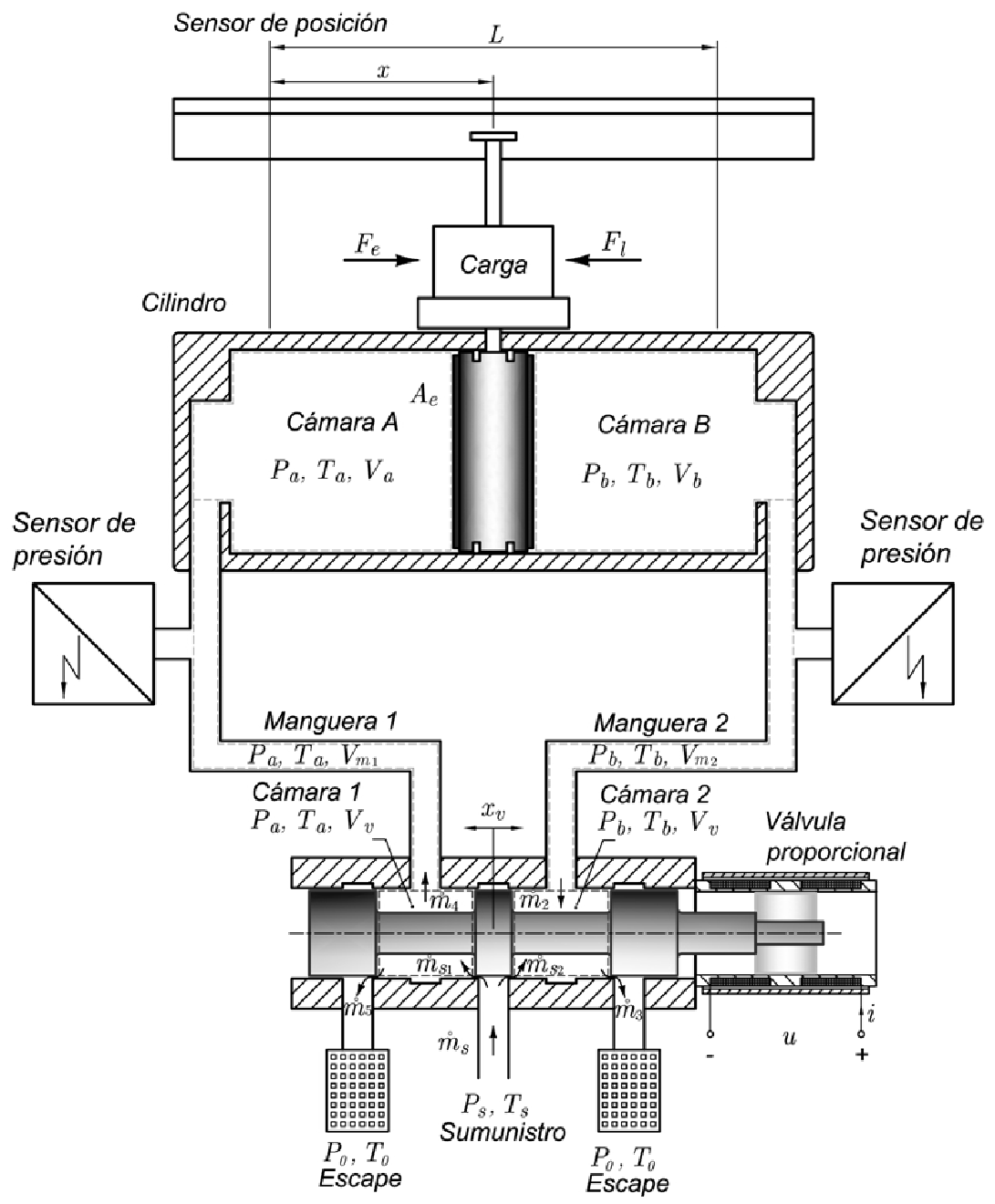

Fig. 1. Servomecanismo neUmático de posicionamiento

La ecuación de la dinámica del émbolo se puede escribir como:

$$
m_{e} \ddot{x}=\left\{\begin{array}{l}
A_{e}\left(P_{a}-P_{b}\right)-F_{f}-F_{l}-F_{i m p}, \quad x<0 \quad o x>L \\
A_{e}\left(P_{a}-P_{b}\right)-F_{f}-F_{l}, 0 \leq x \leq L
\end{array}\right.
$$


donde $A_{e}\left(P_{a}-P_{b}\right)$ es la fuerza efectiva en el émbolo debido a la diferencia de presiones en las cámaras $\mathrm{A}$ y $\mathrm{B}$ del cilindro, $m_{e}$ es la masa del émbolo (incluido el carro del cilindro), $F_{l}$ es la fuerza de la carga externa, $F_{f}$ es la fuerza de fricción (seca + viscosa) y $F_{i m p}$ es la fuerza de impacto en los extremos. El modelo de la fuerza de impacto está basado en la teoría elastoplástica de los materiales:

$$
F_{\text {imp }}=\left\{\begin{array}{l}
k_{e} x+B_{e} \stackrel{\circ}{x}, x<0 \\
k_{e}(x-l)+B_{e} \stackrel{\circ}{x}, x>L
\end{array}\right.
$$

donde $k_{e}$ es la rigidez equivalente y $B_{e}$ es el coeficiente de amortiguación equivalente.

La fricción del sistema está representada por el modelo de LuGre, el cual está definido como:

$$
\begin{aligned}
& \frac{d z}{d t}=v-\frac{\sigma_{0}|v| z}{F_{c}+\left(F_{s}-F_{c}\right) e^{-\left(\frac{v}{v_{s}}\right)^{2}}} \\
& F_{f}=\sigma_{0} z+\sigma_{1} \stackrel{\circ}{z}+B v
\end{aligned}
$$

donde $v=\stackrel{0}{x}$ es la velocidad del émbolo, $F_{s}$ es la fuerza de fricción estática, $F_{c}$ es la fuerza de fricción de Coulomb, $z$ es la deflexión media de cerda, $v_{s}$ es la velocidad de deslizamiento, $\sigma_{0}$ es el coeficiente de rigidez, $\sigma_{l}$ es el coeficiente de amortiguamiento seco y $B$ es el coeficiente de fricción viscosa. La dinámica de la presión en el sistema está descrita por las ecuaciones:

$$
\begin{aligned}
& \frac{d P_{a}}{d t}=\frac{n}{x+x_{a r}+\frac{V_{m_{1}}}{A_{e}}}\left[\frac{R T_{a_{0}} P_{a}^{\frac{n-1}{n}}}{A_{e} P_{a_{0}}^{\frac{n-1}{n}}} \stackrel{\circ}{m}_{4}+P_{a} v\right] \\
& \frac{d P_{b}}{d t}=\frac{n}{L-x+x_{b r}+\frac{V_{m_{2}}}{A_{e}}}\left[\frac{R T_{b_{0}} P_{b}^{\frac{n-1}{n}}}{A_{e} P_{b_{0}}^{\frac{n-1}{n}}} \stackrel{\circ}{m}_{2}+P_{b} v\right]
\end{aligned}
$$


donde $P_{a}$ y $T_{a}$ son la presión y la temperatura absolutas en el volumen de control A (cámara 1 de la válvula, manguera 1 y cámara A del cilindro), $P_{b}$ y $T_{b}$ y son la presión y la temperatura absolutas en el volumen de control B (cámara 2 de la válvula, manguera 2 y cámara $\mathrm{B}$ del cilindro), $V_{a}$ es el volumen de cada cámara de la válvula, $V_{m_{1}}$ y $V_{m_{2}}$ son los volúmenes de las mangueras $1 \mathrm{y} 2$, respectivamente, $x_{a r}$ y $x_{b r}$ son los desplazamientos equivalentes remanentes en los extremos del cilindro, $P_{a_{0}}$ y $T_{a_{0}}$ son los estados iniciales de la presión y la temperatura en el volumen de control A, y son los estados iniciales de la presión y la temperatura en el volumen de control B, $m_{4}$ y $\stackrel{\circ}{2}_{2}$ son los flujos de masa por los puertos de trabajo 4 y 2 de la válvula, $R$ es la constante particular del aire y $n$ es la constante politrópica del proceso.

Los flujos de masa por los puertos de trabajo 4 y 2 se pueden escribir como:

$$
\begin{aligned}
& \stackrel{\circ}{m}_{4}=A_{r s_{1}}(u) f\left(P_{s}, T_{s}, \frac{P_{a}}{P_{s}}\right)-A_{r_{5}}(u) f\left(P_{a}, T_{a}, \frac{P_{0}}{P_{a}}\right) \\
& \stackrel{\circ}{m}_{2}=A_{r_{3}}(u) f\left(P_{b}, T_{b}, \frac{P_{0}}{P_{b}}\right)-A_{r s_{2}}(u) f\left(P_{s}, T_{s}, \frac{P_{b}}{P_{s}}\right)
\end{aligned}
$$

donde $A_{r s 1}$ y $A_{r s 2}$ son las áreas relativas de los orificios de paso en el puerto de suministro $1, A_{r_{3}}$ y $A_{r 5}$ son las áreas relativas de los orificios de paso en los puertos de escape 3 y 5 , respectivamente, y $u$ es la señal de voltaje aplicada a la válvula. La función $f$ se define como:

$$
f\left(P_{u}, T_{u}, \frac{P_{d}}{P_{u}}\right)=\left\{\begin{array}{l}
P_{u} C \rho_{n} \sqrt{\frac{T_{n}}{T_{u}}}, \quad \frac{P_{d}}{P_{u}} \leq b \\
P_{u} C \rho_{n} \sqrt{\frac{T_{n}}{T_{u}}} \sqrt{1-\left(\frac{\frac{P_{d}}{P_{u}}-b}{1-b}\right)^{2}}, \quad b<\frac{P_{d}}{P_{u}} \leq 0.999 \\
k_{1} P_{u}\left(1-\frac{P_{d}}{P_{u}}\right) \sqrt{\frac{T_{n}}{T_{u}}}, \quad \frac{P_{d}}{P_{u}} \geq 0.999
\end{array}\right.
$$


$k_{1}=10^{3} C \rho_{n} \sqrt{1-\left(\frac{0.999-b}{1-b}\right)^{2}}, P_{u}$ y $T_{u}$ son la presión y la temperatura absolutas en la sección de alta presión, $P_{d}$ es la presión absoluta en la sección de baja presión, $\rho_{n}$ y $T_{n}$ son la densidad y la temperatura del aire a las condiciones de referencia establecidas por la norma ISO $6358, b$ es la relación crítica de presiones y $C$ la conductancia sónica.

\section{Puntos de equilibrio}

Con el fin de determinar los puntos de equilibrio del sistema, el modelo matemático se representa por la ecuación de estado:

$$
\stackrel{\circ}{\mathbf{x}}=f(\mathbf{x}, \mathbf{u}), \quad \mathbf{x}\left(t_{0}\right)=\mathbf{x}_{0}
$$

donde $\mathrm{X} \in \mathfrak{R}^{n}$ es el vector de estados en el tiempo $\mathrm{t}, \mathbf{u} \in \mathfrak{R}^{m}$ es el vector de entradas y $f: \mathfrak{R}^{n} \rightarrow \mathfrak{R}^{n}$ es el vector de campo. Puesto que el vector de campo no depende explícitamente del tiempo, el tiempo inicial siempre se toma como $t_{0}=\mathbf{0}$. Así, $x_{1}=P_{a}, x_{2}=P_{b}$, $x_{3}=x, x_{4}=v$ y $x_{5}=z$. Además se tienen en cuenta las siguientes suposiciones generales: Los procesos termodinámicos en los volúmenes de control son isotérmicos, es decir $n=1$, el flujo de aire se encuentra en régimen subsónico y el actuador trabaja sin carga.

Por lo tanto, el sistema de ecuaciones resultante es:

$$
\begin{gathered}
\dot{\circ}_{1}=\frac{\left[\frac{R T_{a o}}{A_{e}}\left(A_{r s_{1}}(u) f\left(P_{s}, T_{s}, \frac{x_{1}}{P_{s}}\right)-A_{r_{5}} f\left(x_{1}, T_{a}, \frac{P_{0}}{x_{1}}\right)\right)-x_{1} x_{4}\right]}{x_{3}+x_{a r}+\frac{V_{m_{1}}}{A_{e}}} \\
\circ_{2}=\frac{\left[-\frac{R T_{a o}}{A_{e}}\left(A_{r_{3}}(u) f\left(x_{2}, T_{b}, \frac{P_{0}}{x_{2}}\right)-A_{r s_{2}} f\left(P_{s}, T_{s}, \frac{x_{2}}{P_{s}}\right)\right)+x_{2} x_{4}\right]}{L-x_{3}+x_{b r}+\frac{V_{m_{2}}}{A_{e}}}
\end{gathered}
$$




$$
\begin{aligned}
& \stackrel{\circ}{x}_{3}=x_{4} \\
& \dot{x}_{4}=\frac{A_{e}\left(x_{1}-x_{2}\right)-\left[\sigma_{0} x_{5}+\sigma_{0}\left(x_{4}-\frac{\sigma_{0}\left|x_{4}\right| x_{5}}{F_{c}+\left(F_{s}-F_{c}\right) e^{-\left(\frac{x_{4}}{v_{s}}\right)^{2}}}\right)+B x_{4}\right]}{m_{e}} \\
& {\stackrel{\circ}{x_{5}}}=x_{4}-\frac{\sigma_{0}\left|x_{4}\right| x_{5}}{F_{c}+\left(F_{s}-F_{c}\right) e^{-\left(\frac{x_{4}}{v_{s}}\right)^{2}}}
\end{aligned}
$$

con

$$
f\left(P_{u}, T_{u}, \frac{P_{d}}{P_{u}}\right)=P u C \rho u \sqrt{\frac{T_{n}}{T_{u}}} \sqrt{1-\left(\frac{\frac{P_{d}}{P_{u}}-b}{1-b}\right)^{2}}
$$

El anterior modelo permite establecer las siguientes situaciones para el análisis del equilibrio del sistema en lazo abierto:

\subsection{Equilibrio del sistema para $x_{4}=0$ y $0<x_{3}<L$}

En este caso se supone que el émbolo del cilindro está empotrado elásticamente, de tal forma que experimenta deslizamiento predeslizamiento (presliding). Igualando (11)-(15) a cero y resolviendo para $x_{1}, x_{2}, x_{3}, x_{4}$ y $x_{5}$ se obtiene analíticamente el equilibrio:

$$
\begin{aligned}
& x_{1}^{e q}=\frac{\delta_{1}+\sqrt{\delta_{2}+\delta_{3}}}{\delta_{4}} \\
& x_{2}^{e q}=\frac{\varepsilon_{1}+\sqrt{\varepsilon_{2}+\varepsilon_{3}}}{\varepsilon_{4}} \\
& x_{3}^{e q}=x_{3} \\
& x_{4}^{e q}=0
\end{aligned}
$$




$$
x_{5}^{e q}=\frac{A e\left(x_{1}^{e q}-x_{2}^{e q}\right)}{\sigma_{0}}
$$

donde

$$
\begin{gathered}
\delta_{1}=b\left(A_{r s_{1}}^{2}(u) P_{s} T_{a_{0}}-A_{r s_{2}}^{2}(u) P_{0} T_{b_{0}}\right) \\
\delta_{2}=\left(b^{2}-2 b+1\right)\left[\left(A_{r s_{1}}(u) P_{s} T_{a_{0}}\right)^{2}-\left(A_{r s_{2}}(u) P_{0} T_{b_{0}}\right)^{2}\right] \\
\delta_{3}=A_{r s_{1}}^{2}(u) A_{r_{5}}^{2}(u) T_{s} T_{a_{0}}\left[P s\left(4 b^{2}-4 b+1\right)+P_{0}^{2}-2 b^{2} P_{0} P_{s}\right] \\
\delta_{4}=A_{r s_{1}}^{2}(u) T_{a_{0}}-(2 b-1) A_{r_{3}}^{2}(u) T_{s}
\end{gathered}
$$

El equilibrio es dependiente del voltaje aplicado a la válvula $u$. La ecuación (18) con $x_{3} \in(0, L)$ demuestra la presencia de un subespacio de equilibrio unidimensional, ya que el estado $x_{3}$ puede asumir cualquier valor dentro de dicho intervalo sin que los demás equilibrios se alteren.

Las curvas de equilibrio teórico (líneas continuas) y las de equilibrio experimental (líneas a trazos) para un intervalo $4.5 \leq$ $u \leq 5.5$. $V$ se muestran en la Fig. 2. El equilibrio para $x_{1}$ y $x_{2}$ no depende de la capacitancia de las cámaras del cilindro, la cual es función del volumen de las mismas, por tanto, el equilibrio para estos estados no depende de la posición fija $x_{3}$ en la que se encuentre el émbolo.

La estabilidad del sistema se puede estimar mediante el método de linealización de Lyapunov donde se determinan los valores propios $\lambda_{1}$ de la matriz Jacobiana evaluada en cada punto de equilibrio. La matriz Jacobiana del sistema está definida por: 

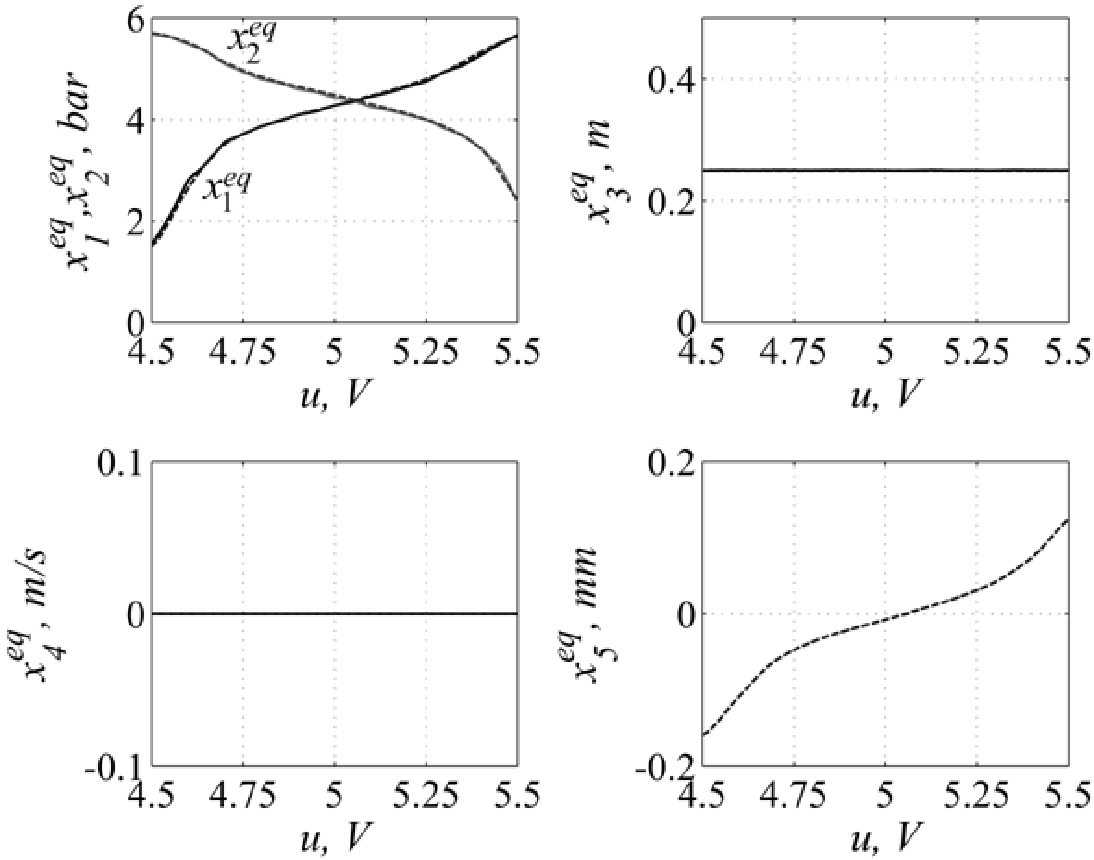

Fig. 2. Equilibrio del Sistema para $4.5 \leq u \leq 5.5, x_{3}=L / 2$

$$
J_{0}=\left[\begin{array}{ccc}
\frac{\partial f_{1}}{\partial x_{1}} & \frac{\partial f_{1}}{\partial x_{2}} \ldots & \frac{\partial f_{1}}{\partial x_{5}} \\
\frac{\partial f_{2}}{\partial x_{1}} & \frac{\partial f_{2}}{\partial x_{2}} \cdots & \frac{\partial f_{2}}{\partial x_{5}} \\
\vdots & \vdots & \vdots \\
\frac{\partial f_{5}}{\partial x_{1}} & \frac{\partial f_{5}}{\partial x_{2}} \cdots & \frac{\partial f_{5}}{\partial x_{5}}
\end{array}\right]\left\{\begin{array}{lllll}
\left.x_{1}^{e q}, x_{2}^{e q}, x_{3}^{e q}, x_{4}^{e q}, x_{5}^{e q}\right\}
\end{array}\right.
$$

Los valores propios se determinan resolviendo el polinomio característico resultante de la expresión:

$$
\operatorname{det}\left(\lambda I-J_{0}\right)=0
$$

En la Fig. 3 se muestra el comportamiento obtenido numéricamente, de la parte real de los autovalores frente al voltaje aplicado $u$, en el intervalo $[4.5,5.5] \mathrm{V}$. 

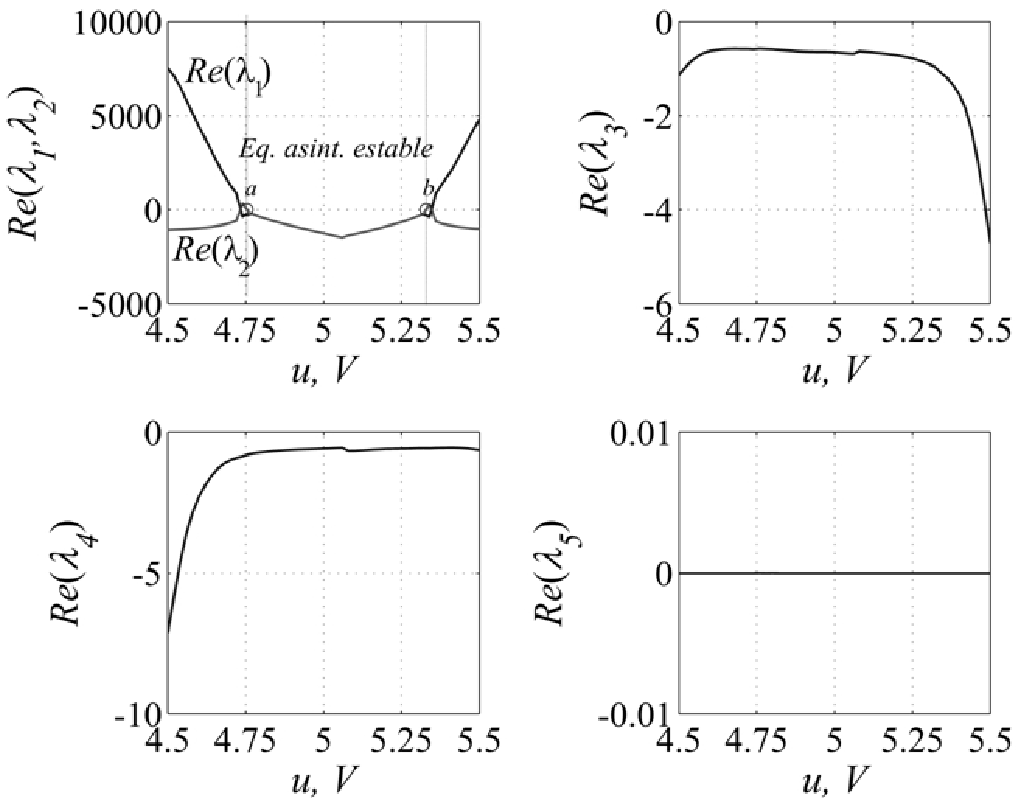

Fig. 3. COMPORTAMIENTO DE LA PARTE REAL DE LOS AUtOVALORES PARA $4.5 \leq u \leq 5.5 V$ Y $x_{3}=L / 2$

A partir la Fig. 3, se puede destacar: La comprobación de la existencia de un subespacio unidimensional de equilibrio ya que $\operatorname{Re}\left(\lambda_{5}\right)=0$ en todo el intervalo; la presencia de una región de equilibrio comprendida en el intervalo $\left[u_{a^{\prime}}, u_{b}\right]=[4.75,5.32] \mathrm{V}$ dentro de la cual el sistema presenta un subespacio de equilibrio unidimensional asistóticamente estable (Slotine, 1991), puesto que $\operatorname{Re}\left(\lambda_{1}, \lambda_{2}, \lambda_{3}\right.$, $\left.\lambda_{4}\right)<0$; fuera de la región de equilibrio, el sistema presenta un subespacio de equilibrio inestable puesto que, en primera instancia, se tiene $\operatorname{Re}\left(\lambda_{2}\right)>0$ y $\operatorname{Re}\left(\lambda_{1}, \lambda_{3}, \lambda_{4}\right)<0$, y posteriormente se tiene $\operatorname{Re}\left(\lambda_{1}\right)>0$ y $\operatorname{Re}\left(\lambda_{2}, \lambda_{3}, \lambda_{4}\right)<0$ y dentro de la región de equilibrio asintóticamente estable es posible determinar el valor de voltaje para el cual se cumple $x_{1}^{e q}=x_{2}^{e q}, x_{3}^{e q}=x_{3}, x_{4}^{e q}=0$ y $x_{5}^{e q}=0$ igualando entre sí (11) y (12) y resolviendo para $u$, de donde se obtiene un valor $u^{*}=5.055 V$ que corresponde al voltaje en el cual la corredera de la válvula se sitúa en la posición central. 


\subsection{Equilibrio en los extremos $x_{3}=0$ y $x_{3}=L$}

\subsubsection{Equilibrio en $x_{3}=0$}

En este extremo de la carrera del cilindro está presenta la fuerza de impacto $F_{i m p}=k_{e} x_{3}+B_{e} x_{3}$, la cual modifica únicamente (14) quedando:

$$
\dot{x}_{4}=\frac{A_{e}\left(x_{1}-x_{2}\right)-\left[\sigma_{0} x_{5}+\sigma_{0}\left(x_{4}-\frac{\sigma_{0}\left|x_{4}\right| x_{5}}{F_{c}+\left(F_{s}-F_{c}\right) e^{-\left(\frac{x_{4}}{v_{s}}\right)^{2}}}\right)+B x_{4}+F_{i m p}\right]}{m_{e}}
$$

de esta manera, el sistema resultante se expresa como:

$$
\begin{gathered}
A_{r s_{1}}(u) f\left(P_{s}, T_{s}, \frac{x_{1}}{P_{s}}\right)-A_{r_{5}} f\left(x_{1}, T_{a}, \frac{P_{0}}{x_{1}}\right)=0 \\
A_{r_{3}}(u) f\left(x_{2}, T_{b}, \frac{P_{0}}{x_{2}}\right)-A_{r s_{2}} f\left(P_{s}, T_{s}, \frac{x_{2}}{P_{s}}\right)=0 \\
A_{e}\left(x_{1}-x_{2}\right)-\sigma_{0} x_{5}-k_{e} x_{3}=0 \\
x_{4}-\frac{\sigma_{0}\left|x_{4}\right| x_{5}}{F_{c}+\left(F_{s}-F_{c}\right) e^{-\left(\frac{x_{4}}{v_{s}}\right)^{2}}}=0
\end{gathered}
$$

de (27) se deduce:

$$
x_{5}=\frac{F_{c}+\left(F_{s}-F_{c}\right) e^{-\left(\frac{x_{4}}{v_{s}}\right)^{2}}}{\sigma_{0}} \frac{x_{4}}{\left|x_{4}\right|}
$$

si $x_{2}>x_{1}$, se establece:

$$
x_{5}^{e q}=\lim _{x_{4} \rightarrow 0^{-}} \frac{F_{c}+\left(F_{s}-F_{c}\right) e^{-\left(\frac{x_{4}}{v_{s}}\right)^{2}}}{\sigma_{0}} \frac{x_{4}}{\left|x_{4}\right|}=-\frac{F s}{\sigma_{0}}=\frac{F_{s_{n}}}{\sigma_{0}}
$$


por lo tanto, el equilibrio del sistema en el extremo izquierdo se puede escribir como:

$$
\begin{gathered}
x_{1}^{e q}=\frac{\delta_{1}+\sqrt{\delta_{2}+\delta_{3}}}{\delta_{4}} \\
x_{2}^{e q}=\frac{\varepsilon_{1}+\sqrt{\varepsilon_{2}+\varepsilon_{3}}}{\varepsilon_{4}} \\
x_{3}^{e q}=\frac{A_{e}\left(x_{1}-x_{2}\right)+F_{s_{n}}}{k_{e}} \\
x_{4}^{e q}=0 \\
x_{5}^{e q}=\frac{F_{s_{n}}}{\sigma_{0}}
\end{gathered}
$$
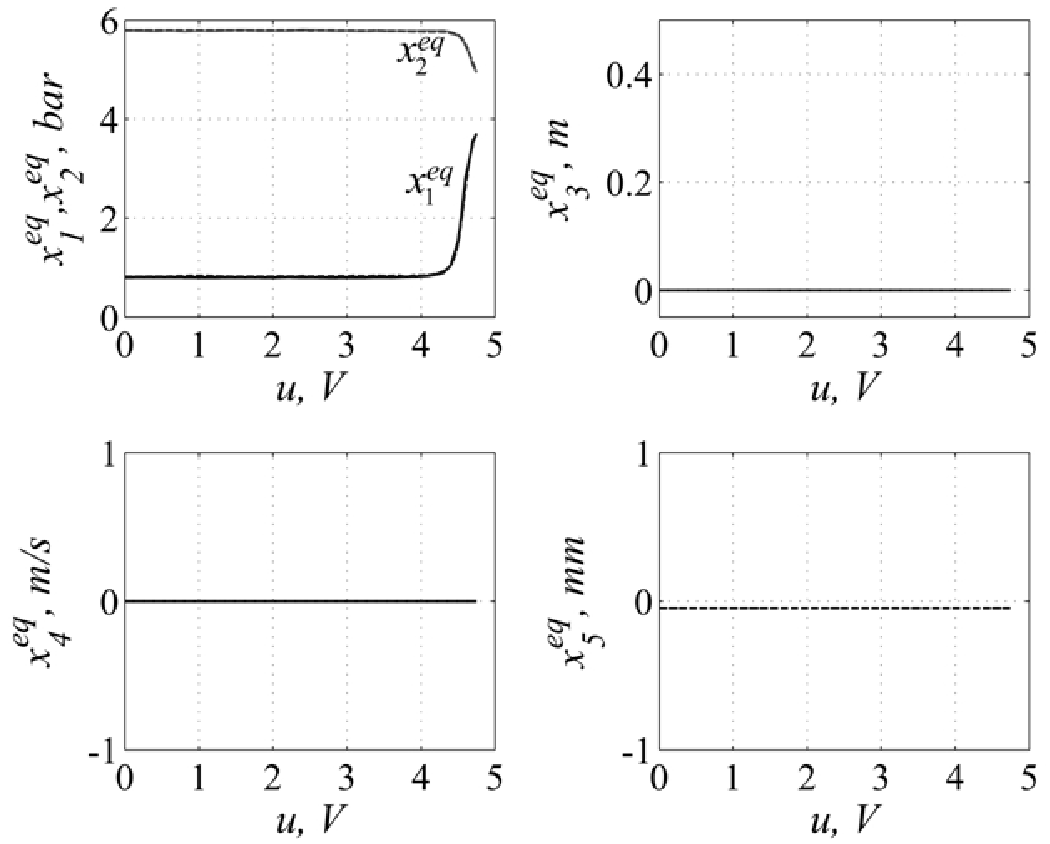

FIG. 4. EQUILIBRIO PARA $0 \leq u \leq u_{a} \curlyvee x_{3}=0$ 
El equilibrio teórico y experimental para $x_{3}=0$ se muestra en la Fig. 4. Numéricamente se determina que el sistema presenta un subespacio de equilibrio asintóticamente estable dentro del intervalo $0 \leq u \leq u_{a}$, puesto que $\operatorname{Re}\left(\lambda_{1}, \lambda_{2}, \lambda_{3}, \lambda_{4}\right)<0$ y $\operatorname{Re}\left(\lambda_{5}\right)=0$, tal como se ilustra en la Fig. 5.
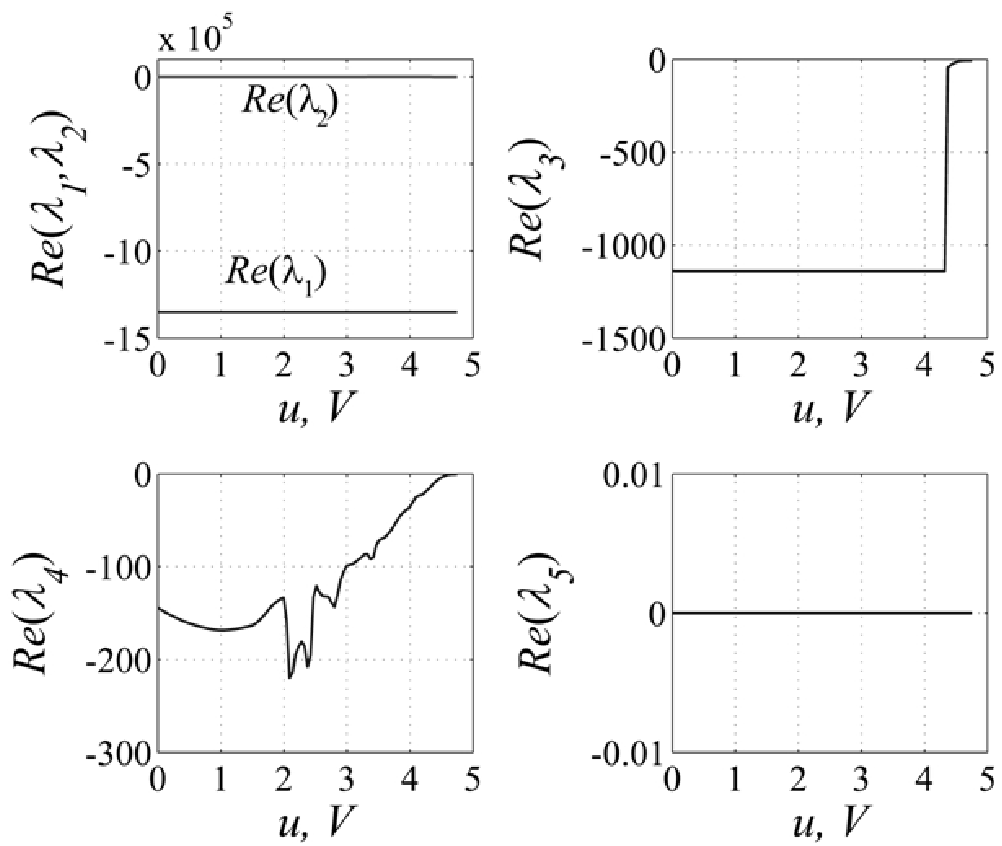

Fig. 5. Comportamiento de LA PARTE REAL DE LOS AUtOVAloRes

$$
\text { PARA } 0 \leq u \leq u_{a} \text { Ү } x_{3}=0
$$

\subsubsection{Equilibrio en $x_{3}=L$}

En el extremo $x_{3}=L$ la fuerza de impacto es $F_{i m p}=k_{e}\left(L-x_{3}\right)+$ $B_{e} x_{3}$. Por tanto, el sistema de ecuaciones de equilibrio resultante es:

$$
A_{r s_{1}}(u) f\left(P_{s}, T_{s}, \frac{x_{1}}{P_{s}}\right)-A_{r_{s}} f\left(x_{1}, T_{a}, \frac{P_{0}}{x_{1}}\right)=0
$$




$$
\begin{gathered}
A_{r_{3}}(u) f\left(x_{2}, T_{b}, \frac{P_{0}}{x_{2}}\right)-A_{r_{2}} f\left(P_{s}, T_{s}, \frac{x_{2}}{P_{s}}\right)=0 \\
A_{e}\left(x_{1}-x_{2}\right)-\sigma_{0} x_{5}-k_{e}\left(x_{3}-L\right)=0 \\
x_{4}-\frac{\sigma_{0}\left|x_{4}\right| x_{5}}{F_{c}+\left(F_{s}-F_{c}\right) e^{-\left(\frac{x_{4}}{v_{s}}\right)^{2}}}=0
\end{gathered}
$$

Si $x_{3}>x_{2}$ se establece:

$$
x_{5}^{e q}=\lim _{x_{4} \rightarrow 0^{+}} \frac{F_{c}+\left(F_{s}-F_{c}\right) e^{-\left(\frac{x_{4}}{v_{s}}\right)^{2}}}{\sigma_{0}} \frac{x_{4}}{\left|x_{4}\right|}=\frac{F_{s}}{\sigma_{0}}=\frac{F_{s_{p}}}{\sigma_{0}}
$$

de esta manera, el equilibrio del sistema en el extremo derecho se puede escribir como:

$$
\begin{gathered}
x_{1}^{e q}=\frac{\delta_{1}+\sqrt{\delta_{2}+\delta_{3}}}{\delta_{4}} \\
x_{2}^{e q}=\frac{\varepsilon_{1}+\sqrt{\varepsilon_{2}+\varepsilon_{3}}}{\varepsilon_{4}} \\
x_{3}^{e q}=\frac{A e(x 1-x 2)-F_{s_{p}}}{k_{e}} \\
x_{4}^{e q}=0 \\
x_{5}^{e q}=\frac{F_{s_{p}}}{\sigma_{0}}
\end{gathered}
$$

El equilibrio teórico y experimental para $x_{3}=L$ se muestra en la Fig. 6. De igual manera que en el caso anterior, numéricamente se determina que el sistema presenta un subespacio de equilibrio asintóticamente estable dentro del intervalo $u_{b}<u \leq 10 \mathrm{~V}$, puesto que $\operatorname{Re}\left(\lambda_{1}, \lambda_{2}, \lambda_{3}, \lambda_{4}\right)<0$ y $\operatorname{Re}\left(\lambda_{5}\right)=0$. La Fig. 7 muestra estos resultados. 

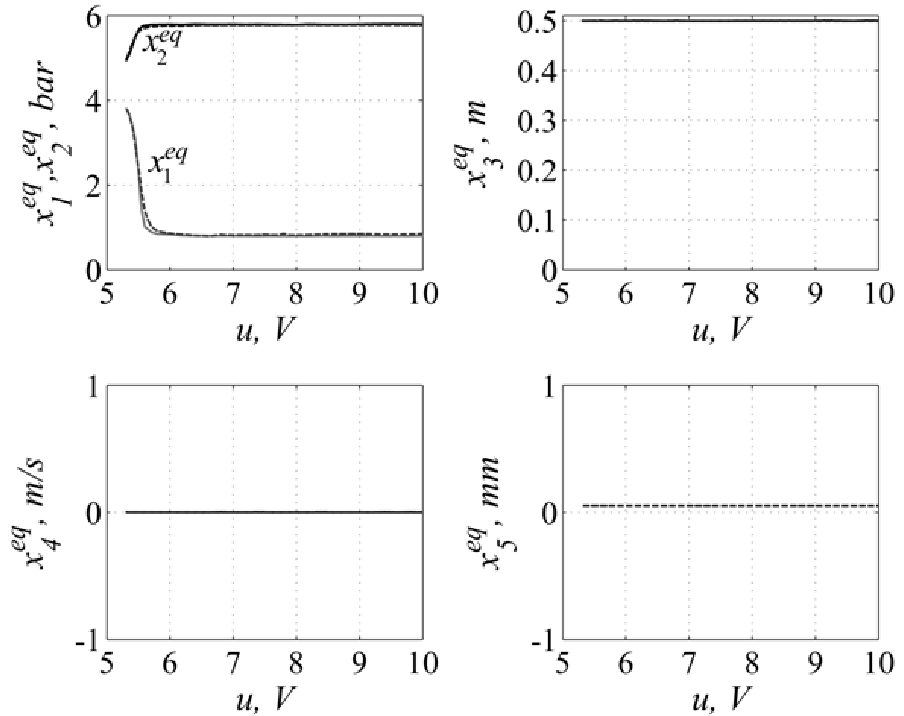

FIG. 6. EQUILIBRIO PARA $u_{b}<u \leq 10$ y $x_{3}=L$
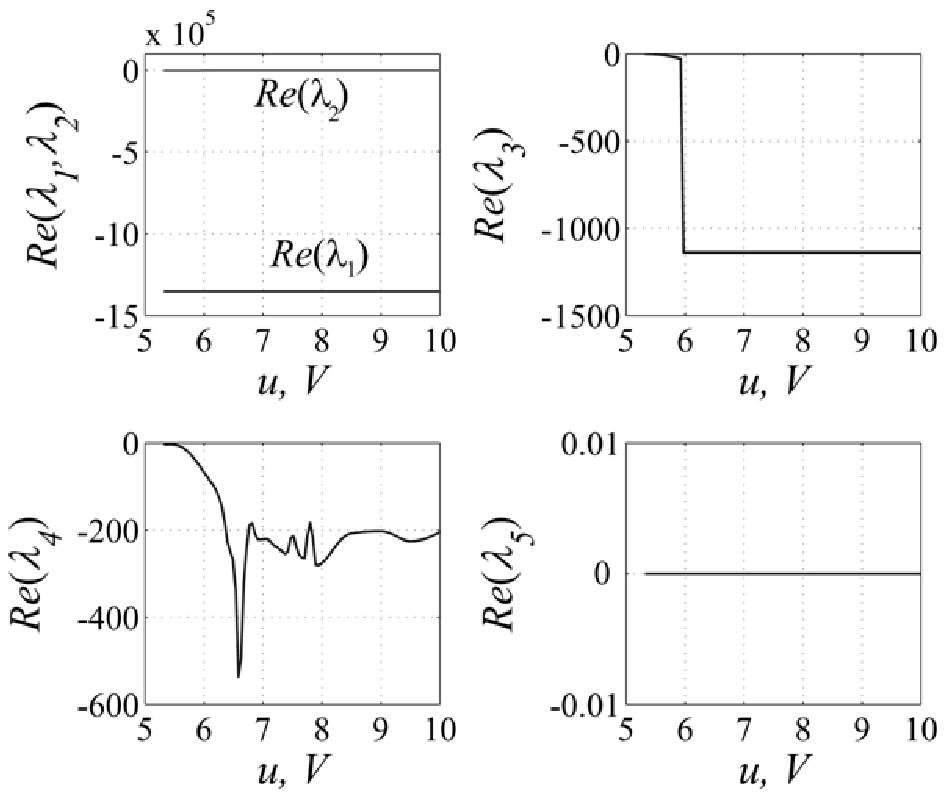

Fig. 7. Comportamiento de LA PARTE REAL DE LOS AUtOValores PARA $u_{b}<u \leq 10$ y $x_{3}=L$ 
3.3 Equilibrio del sistema para $x_{4} \neq 0\left(o x_{4}=0\right)$ y $0<x_{3}<L$

Para esta situación, se considera que el émbolo puede desplazarse a lo largo de la carrera del cilindro pero también puede detenerse en cualquier punto. El equilibrio del sistema se determina a partir de las ecuaciones:

$$
\begin{aligned}
& \frac{R T_{a_{0}}}{A_{e}}\left[A_{r s_{1}}(u) f\left(P_{s}, T_{s}, \frac{x_{1}}{P_{s}}\right)-A_{r_{5}} f\left(x_{1}, T_{a}, \frac{P_{0}}{x_{1}}\right)\right]-x_{1} x_{4}=0 \\
& -\frac{R T_{b_{0}}}{A_{e}}\left[A_{r_{3}}(u) f\left(x_{2}, T_{b}, \frac{P_{0}}{x_{2}}\right)-A_{r s_{2}} f\left(P_{s}, T_{s}, \frac{x_{2}}{P_{s}}\right)\right]+x_{2} x_{4}=0 \\
& A_{e}\left(x_{1}-x_{2}\right)-\left[F_{c}+\left(F_{s}-F_{c}\right) e^{-\left(\frac{x_{4}}{v_{s}}\right)^{2}}\right] \operatorname{sign}\left(x_{4}\right)-B x_{4}=0
\end{aligned}
$$
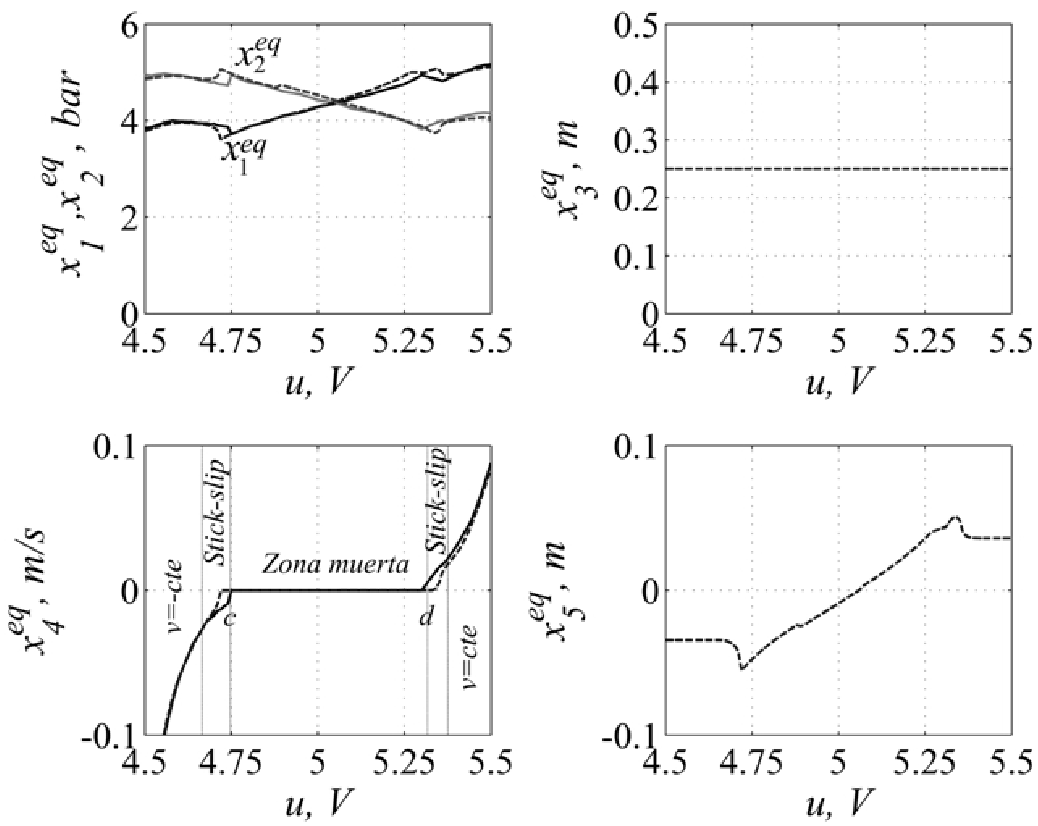

FIG. 8. EQUILIBRIO PARA $x_{4} \neq 0$ 
El equilibrio del sistema obtenido numéricamente en el intervalo $4.5 \leq u \leq 5.5, V$ frente al equilibrio obtenido experimentalmente, se muestra en la Fig. 8. Según esta figura, el equilibrio $x_{4}^{e q}$ indica la presencia de una zona muerta debida a la fricción del sistema, establecida dentro del intervalo de voltaje $\left[u_{c}, u_{d}\right]=[4.743,5.337] \mathrm{V}$. En la zona muerta, la fuerza resultante debido a la diferencia de presiones no supera a la fuerza de fricción estática existente entre el émbolo y la camisa del cilindro, por tanto, el émbolo permanece estático. Puesto que $x_{4}^{e q}=0$, el sistema presenta un subespacio de equilibrio en donde el equilibrio para $x_{1}, x_{2}, x_{4}$ y $x_{5}$ no depende de $x_{3}$.

La zona muerta presenta un subespacio de equilibrio asintóticamente estable ya que $\operatorname{Re}\left(\lambda_{1}, \lambda_{2}, \lambda_{3}, \lambda_{4}\right)<0$ y $\operatorname{Re}\left(\lambda_{5}\right)=0$, hecho indicado en la Fig. 9. Fuera de la zona muerta el equilibrio $x_{4}^{e q}$ presenta dos regiones donde el émbolo se desplaza a velocidad constante en un sentido y en el otro. Es de importancia destacar que esta velocidad no depende de la posición inicial de donde parte
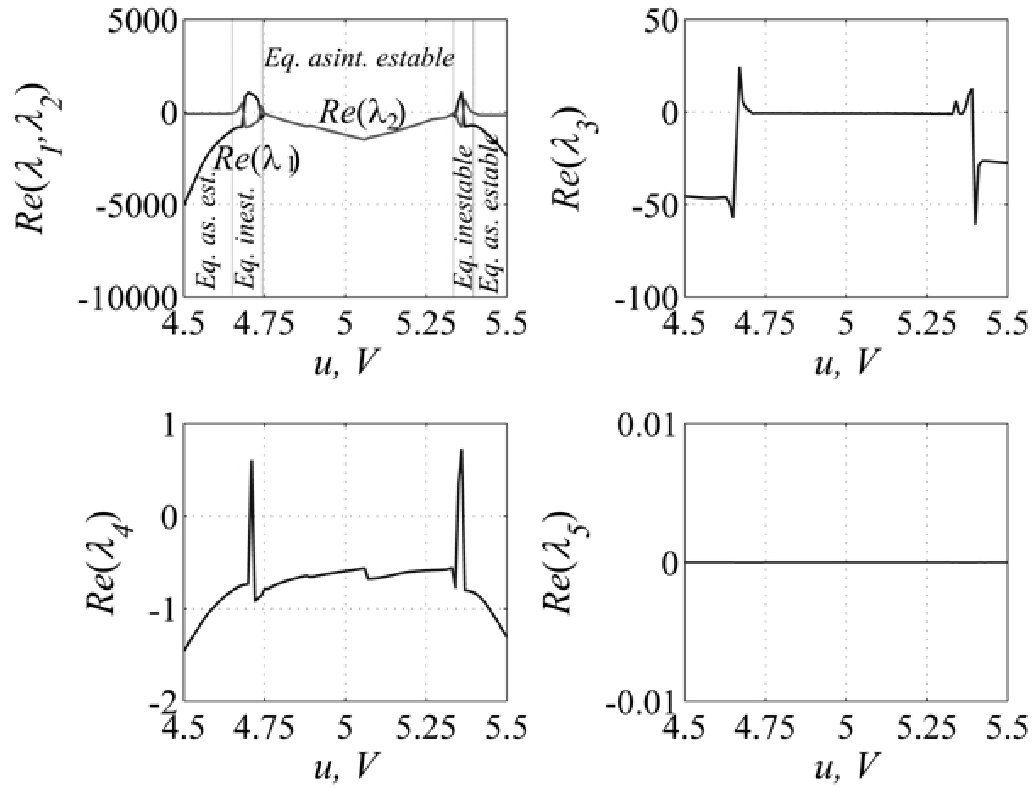

Fig. 9. Comportamiento de La parte ReAl de los autovalores para $x_{4} \neq 0$ 
el émbolo. En esta dos regiones, el sistema exhibe un subespacio de equilibrio asintóticamente estable puesto que $\operatorname{Re}\left(\lambda_{1}, \lambda_{2}, \lambda_{3}, \lambda_{4}\right)<0$ y $\operatorname{Re}\left(\lambda_{5}\right)=0$. Existe una zona de transición entre la zona muerta y las regiones donde el émbolo se desplaza a velocidad constante, en la cual se presenta el fenómeno no-lineal denominado adherenciadeslizamiento (stick-slip).

\subsection{Fenómeno adherencia-deslizamiento (stick-slip)}

Este fenómeno es un comportamiento típico de los sistemas que tienen fricción. Se debe al hecho que la fricción es mayor en el reposo que durante el movimiento. Se caracteriza por la presencia de oscilaciones autoexcitadas alrededor de un equilibrio inestable. En el servosistema neumático el fenómeno adherencia deslizamiento se manifiesta en las regiones de transición de la zona muerta $\left(x_{4}^{e q}=0\right)$ a las regiones donde el émbolo se desplaza con velocidad constante $\left(x_{4}^{e q}=\right.$ cte). Estas regiones se encuentran delimitadas por los cruces por cero que presentan las partes reales de los autovalores complejos conjugados, dándole el carácter oscilatorio al fenómeno.

En la Fig. 10 se muestra el comportamiento de la parte real de los autovalores en las regiones de adherencia-deslizamiento. Según la Fig. 10(a), la región de adherencia-deslizamiento a la izquierda de de la zona muerta se encuentra comprendida en el intervalo $4.662<u<4.743, V$. En los dos límites de este intervalo, $\operatorname{Re}\left(\lambda_{2}\right)$ y $\operatorname{Re}\left(\lambda_{3}\right)$ cruzan simultáneamente el cero. Dentro del intervalo, $\operatorname{Re}\left(\lambda_{1}\right)$ y $\operatorname{Re}\left(\lambda_{2}\right)$ se intercambien alrededor de cero, de igual forma lo hacen $\operatorname{Re}\left(\lambda_{3}\right)$ y $\operatorname{Re}\left(\lambda_{4}\right)$, y $\operatorname{Re}\left(\lambda_{5}\right)$ queda invariante. A partir de este comportamiento se puede deducir que en dicho intervalo siempre existen por lo menos dos autovalores con parte real positiva, por tanto, el equilibrio presente allí es inestable. Según la Fig. 10(b), la región de adherencia-deslizamiento a la derecha de la zona muerta está comprendida en el intervalo 5.337 $<u<5.391, V$ donde nuevamente $\operatorname{Re}\left(\lambda_{2}\right)$ y $\operatorname{Re}\left(\lambda_{3}\right)$ cruzan el cero en sus límites. Dentro de este intervalo se observa el mismo patrón de comportamiento de la parte real de los autovalores $\lambda_{1}, \lambda_{2}, \lambda_{3}, \lambda_{4}$ 
y $\lambda_{5}$ que en la región izquierda, de esta forma se puede inferir que el equilibrio de la región de adherencia-deslizamiento derecha es también inestable.
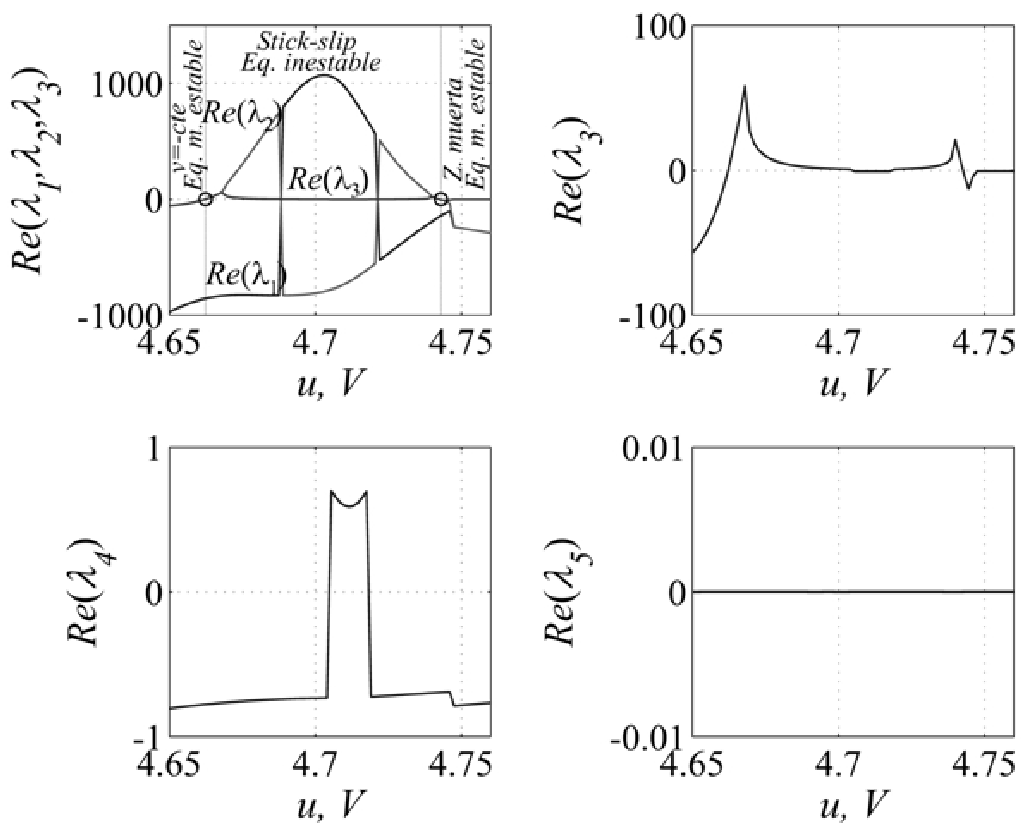

a)

Fig. 10. Comportamiento de Los autovalores En LAS REgIONES DE ADHERENCIAdeslizamiento, A) Región IZquierda y B) Región deRECHA 

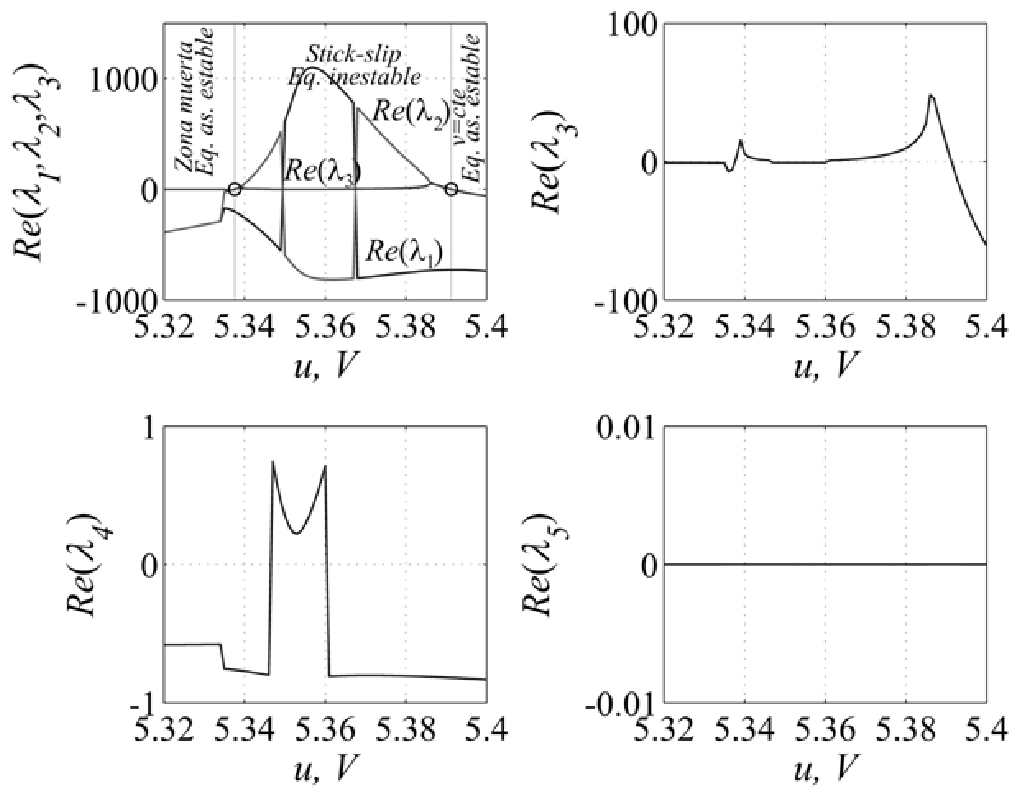

b)

Fig. 10. Comportamiento de los autovalores en LAS REgiones DE ADHERENCIADEsLizAMIENTO, A) REgIÓN IZQUIERDA Y B) REgIón DERECHA

\subsection{Bifurcaciones en lazo abierto}

Tal como se había tratado antes, en la situación de equilibrio en la que el émbolo puede desplazarse a velocidad constante pero también pude detenerse en cualquier posición, el servosistema neumático presenta en lazo abierto dos transiciones en su comportamiento dinámico en estado estacionario. Tomando como parámetro de referencia el voltaje $u$, a medida que se aumenta este voltaje a partir del voltaje para la posición central de la válvula $u^{*}$, el sistema experimenta la primera transición al pasar de una zona muerta a una región donde sucede el fenómeno adherenciadeslizamiento, y posteriormente, la segunda transición se presenta al pasar de la región de adherencia-deslizamiento a una región donde el sistema se desplaza a una velocidad constante. 
Este comportamiento se visualiza en la Fig. 11 donde se muestra el retrato de fases $x_{4}-x_{3}$ para valores del voltaje de referencia $u$, dentro de la zona muerta, en la zona de adherencia-deslizamiento y en la zona donde se desplaza a una velocidad constante, partiendo de un estado inicial donde $x_{1_{0}}<x_{2_{0}}, x_{3_{0}}=\frac{L}{5}, x_{4_{0}}=0$ y $x_{5_{0}}=0$

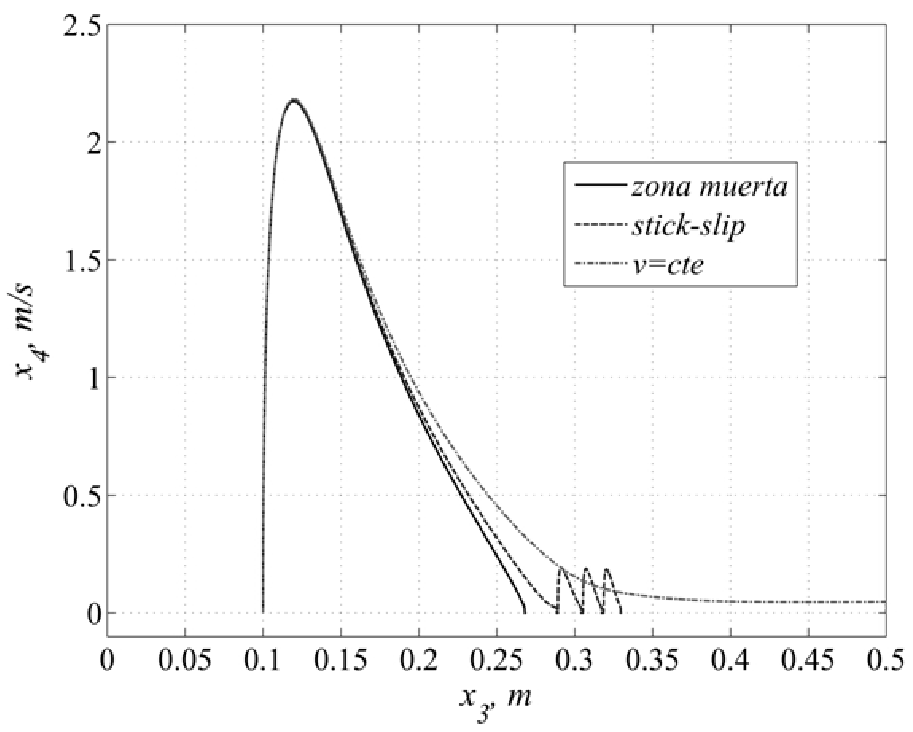

Fig. 11. Retrato de fases $x_{4}-x_{3} \operatorname{CoN} x_{10}<x_{2_{0}}$

Como se puede observar, es claro que en las tres regiones los flujos no son topológicamente equivalentes, lo cual sugiere la existencia de bifurcaciones en los puntos de transición entre las diferentes regiones. Según el análisis de los autovalores en las vecindades de los puntos de transición, la bifurcación que se puede determinar es del tipo Hopf. En este caso la bifurcación de Hopf se manifiesta por el cruce por cero de $\operatorname{Re}\left(\lambda_{2}\right)$ y $\operatorname{Re}\left(\lambda_{3}\right)$ en los límites de las regiones de adherencia-deslizamiento. Como se había analizado anteriormente, dentro de estas regiones el sistema tiene un equilibrio inestable y su comportamiento dinámico se caracteriza por la presencia de oscilaciones periódicas. 
La Fig. 12 ilustra el comportamiento de los autovalores en los puntos críticos (cruces por cero) que delimitan la región de adherencia-deslizamiento, en donde se comprueba la presencia de bifurcaciones de tipo Hopf.

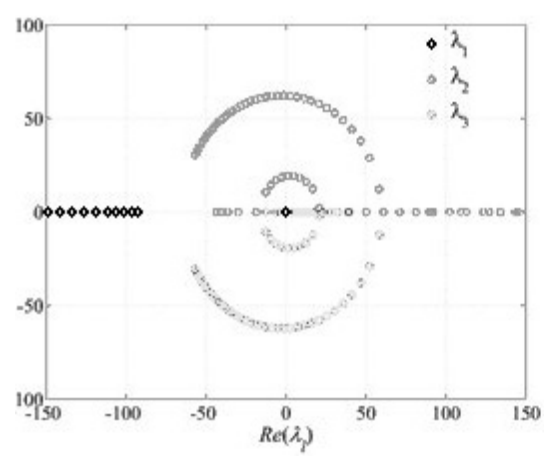

(a)

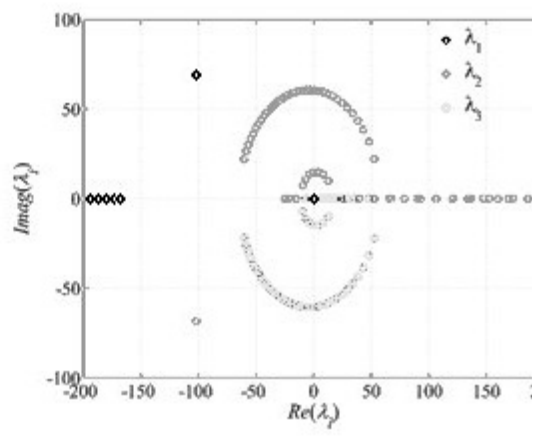

(b)

Fig. 12. Comportamiento autovalores en REgIONES ADHERENCIA-DESLIZAMIENTO;

A) REgIÓN IZQUIERDA Y B) REGIÓN DERECHA

\section{Conclusiones}

A pesar de que la teoría sobre subespacios de equilibrio no está completamente desarrollada y por lo tanto se carece de herramientas analíticas para su tratamiento, numéricamente se pudo establecer en este trabajo que el servomecanismo neumático de posicionamiento presenta en lazo abierto un subespacio unidimiensional de equilibrio que se mantiene a lo largo de todo el rango de la señal de entrada . Este subespacio es asintóticamente estable en la zona muerta y en las regiones donde el émbolo se desplaza a velocidad constante.

Fuera de la zona muerta y las regiones de velocidad constante el sistema presenta dos regiones donde ocurre el fenómeno adherencia-deslizamiento, en estas regiones el equilibrio del sistema es inestable. El fenómeno de adherencia-deslizamiento se caracteriza por que el émbolo se desplaza en forma intermitente generando oscilaciones periódicas en su velocidad, la frecuencia 
de estas oscilaciones depende del voltaje aplicado, disminuyendo cuando el voltaje se acerca a los límites de la zona muerta y aumentando cuando el voltaje se acerca a los límites de las regiones de velocidad constante.

El análisis de los autovalores en los puntos críticos que delimitan las regiones de adherencia-deslizamiento mostró un cambio en la topología dinámica del servosistema en estado estacionario, este cambio topológico puede clasificarse como una bifurcación de tipo Hopf.

\section{Nomenclatura}

$P_{s}, T_{s}:$ Condiciones de suministro, bar, $\mathrm{K}$.

$P_{0}, T_{0}$ : Condiciones atmosféricas, bar, K. $P_{a}, P_{b}$ : Presiones absolutas dentro de los volúmenes de control A y $\mathrm{B}$, respectivamente, bar.

$T_{a}, T_{b}$ : Temperaturas absolutas dentro de los volúmenes de control A y B, respectivamente, $\mathrm{K}$.

$P_{a_{0}}, P_{b_{0}}$ : Presiones absolutas iniciales dentro de los volúmenes de control A y B, respectivamente, $\mathrm{K}$.

$T_{a_{0}}, T_{b_{0}}$ : Temperaturas absolutas iniciales dentro de los volúmenes de control A y B, respectivamente, $\mathrm{K}$.

$x$ : Desplazamiento del émbolo, $\mathrm{m}$.

$v$ : Desplazamiento del émbolo, $\mathrm{m} / \mathrm{s}$.

$\stackrel{\circ}{m}_{s:}$ Flujo de masa de suministro hacia la cámara 1 de la válvula, $\mathrm{kg} / \mathrm{s}$.

$\stackrel{\circ}{m}_{s_{2}:}$ Flujo de masa de suministro hacia la cámara 2 de la válvula, $\mathrm{kg} / \mathrm{s}$.

$\dot{m}_{a}$ : Flujo de masa a través de la superficie de control de la cámara A del cilindro, $\mathrm{kg} / \mathrm{s}$.

$\stackrel{\circ}{m}_{b}$ : Flujo de masa a través de la superficie de control de la cámara $\mathrm{B}$ del cilindro, $\mathrm{kg} / \mathrm{s}$.

$\stackrel{\circ}{m}_{2}$ : Flujo de masa a través del puerto de trabajo 2 de la válvula, $\mathrm{kg} / \mathrm{s}$.

$\stackrel{\circ}{m}_{4}$ Flujo de masa a través del puerto de trabajo 4 de la válvula, $\mathrm{kg} / \mathrm{s}$. 
$A_{r}$ : Área relativa, adimensional.

$\alpha, \beta$ : Relaciones de flujo de masa, adimensionales.

$n$ : Constante politrópica, adimensional.

$A_{e}$ : Área efectiva del émbolo, $\mathrm{m}^{2}$.

$x_{a r}, x_{b r}$ : Desplazamientos remanentes en las cámaras A y B del cilindro, respectivamente, $\mathrm{m}$.

$V_{m_{1}}, V_{m_{2}}$ : Volúmenes de las mangueras 1 y 2 , respectivamente, $\mathrm{m}^{3}$.

$V_{v}$ : Volumen en cada cámara de la válvula, $\mathrm{m}^{3}$.

$L$ : Carrera del cilindro, $\mathrm{m}$.

$m_{e}:$ Masa del émbolo, $\mathrm{kg}$.

$F_{f}$ Fuerza de fricción, N.

$z$ : Deflexión media de cerda, $\mathrm{m}$.

$F_{s}:$ Fuerza de fricción estática, N.

$B$ : Coeficiente de fricción viscosa, $\mathrm{Ns} / \mathrm{m}$.

$\sigma_{0}:$ Coeficiente de rigidez, $\mathrm{N} / \mathrm{m}$.

$\sigma_{i}$ : Coeficiente de fricción seca, $\mathrm{Ns} / \mathrm{m}$.

$v_{s}$ : Velocidad de Stribeck, $\mathrm{m} / \mathrm{s}$.

$P_{u}$ : Presión de alta, bar.

$P_{d}$ : Presión de baja, bar.

$C$ : Conductancia sónica, $\mathrm{m}^{3} / \mathrm{s}$.bar.

$b$ : Relación de presiones crítica, adimensional.

$x_{u}$ : Sobretraslape, $\mathrm{m}$.

$u$ : Voltaje aplicado a la válvula, V.

Notación de Variables de Estado

$\mathbf{x}$ : Vector de estado.

u: Vector de entrada.

$\mathbf{x}$ : Derivada del vector de campo.

J: Matriz Jacobiana.

$\lambda_{1}$ : Valor propio o autovalor su i.

\section{Referencias}

Alves L.H., (2006); Sistemas Dinâmicos. Livraria da Física, São Paulo, 2th edition. 
Canudas de Wit, C., Olsson, H., Åström, K.J., (1995); A New Model for Control of Systems with Friction, IEEE/ASME Transactions on Automatic Control, 40, 419-425.

Chiang, H.D., Flueck, A.J., Shah, K.S., Balu, N., (1995); CPFLOW: A Practical Tool for Tracing System Steady-State Stationary Bahavior Due to Land and Generation Variations, IEEE Transactions on Power Systems, 10, 623-633.

Han J., Sun, Y., Wnag, Y., (2007); Parameter Identification of LuGre Tire Model for the Simplified Motion Dynamics of a Quarter-vehicle Model Based on Ant Colony Algorithm, IEEE Conference on Automation and Logistics, 3039-3043.

Hayashi, S., (2000); Nonlinear Phenomena In Hydraulic Systems, Tohoku University, Sendai, Japan.

Hilborn, R.C., (2000); Chaos and Nonlinear Dynamics, Oxford University Press, Second edition.

Hoppensteadt, F.C., (2000); Analysis and Simulation of Chaotic Systems, Springer, second edition.

Kang, W., Gu, G., Sparks, A., Banda, S., (1999); Bifurcation Test Functions and Surge Control for Axial Flow Compressors, Automatica, 35(2), 229239 .

Kusnetsov, Y., (2004); Elements of Applied Bifurcation Theory, Springer.

Lischinsky, P., Canudas, C., Morel, G., (1999); Friction Compensation for an Industrial Hydraulic Robot, IEEE Control Systems, 19(1), 25-32.

Owen, W., Croft, E., McFarlene, J., (2003); Reducing Stick-Slip Friction in Hydraulic Actuators, IEEE/ASME Transactions on Mechatronics, 8, 362-371.

Parker, T.S., Chua, L.O., (1990); Practical Numerical Algorithms for Chaotic Systems, Springer-Verlag.

Sanders, J.A., Verhulst, F., (1985); Averaging Methods in Nonlinear Dynamical Systems, Springer.

Sastry, S., (1999); Nonlinear Systems, Springer, First edition.

Slotine, J.J.E., Li, W., (1991); Applied Nonlinear Control, Prentice Hall.

Takahiro, K., Manabu, S., (2000); An Analytical and Experimental Study of Chaotic Oscillation in a Pneumatic Cylinder, 1st FPNI-PhD Symp., Hamburg, 303-310. 
Watanabe, M., Mitani, Y., Tsuji, K., (2004); A numerical Method to Evaluate Power System Global Stability Determined by Limit Cycle, IEEE Transactions of Power Systems, 19(4), 1925-1934.

Wiggins, S., (2003); Introduction to Applied Nonlinear Dynamical Systems and Chaos Second, Springer, Second edition.

Yaunjay, W., Jianxie, H., Yazhua, Z., Fengkun, Z., (2008); Analysis and Quenching of Limit Cycles of Electro-Hydraulic Servovalve Control Systems with Friction and Interval Transport Lag, 27th Chinese Control Conference, 212-216. 